\title{
Heterogeneous thermograms: the methods of attack
}

\author{
by B. Vainer and A. Moskalev
}

Rzhanov Institute of Semiconductor Physics, Russian Academy of Sciences, Siberian Branch, Novosibirsk, Russia

\begin{abstract}
Pronouncedly inhomogeneous thermograms occur universally in infrared imaging applications. The heterogeneity as a separate characteristic of a $2 \mathrm{D}$ pattern merits detailed consideration and quantitative estimation. The present contribution is among the first works dedicated to solving this problem. In addition to the review and summarizing of previous results, a new computational method is developed for quantitative estimation of the thermal pattern inhomogeneity. Artificial and really measured thermograms are analyzed with the designed original software. An adequacy of the new method is confirmed.
\end{abstract}

\section{Introduction}

Infrared thermography (IRT) is a well-known experimental method of registration, visualization, and quantitative representation of numerous thermal phenomena encountered in industry, science, and many other fields. It has long been in use in medicine as an effective tool for regional and peripheral blood flow monitoring. This capacity of IRT enables physicians to investigate the action of pharmaceuticals [1-3], identify Raynaud's syndrome, Raynaud's phenomenon, diabetic microangiopathy [4, 5], and some other prevailing pathologies and physiological reactions accompanied by vascular-conditioned manifestations. An increase in blood inflow or, vice versa, reduction of blood inflow caused by arterial lumen narrowing (pathological occlusion) results in an increase or decrease in tissue temperature, respectively. An infrared camera serves for registration of infrared radiation and subsequent computer-aided conversion of this radiation from the infrared to the visible form displayed on a monitor screen in real time. Eventually, the change in temperature of a tissue is represented by the so-called "thermogram," which is a two-dimensional pattern of local temperatures spatially distributed within the infrared camera field of view. The values of temperature are usually evaluated on the basis of the known emissivity of the surface under study. It is commonly accepted that the emissivity of human skin is approximately equal to unity.

Today's challenge is to elevate the artificial intelligence of infrared-imaging-oriented computer systems. Among the actions attacking this problem, of fundamental importance are the search for new methods aimed at mathematical processing as well as computer analysis of thermograms, and the development of these methods to fit the highly sensitive new-generation infrared cameras now available. In the search for objective signs of human organism deviation from the norm, quantitative results obtained from such treatment of measured data, as well as analytical findings made on the basis of these results by computer-based means, could bring down the impact of subjectivity on infrared data interpretation.

Conventional medical infrared diagnostics is mainly based on the search for asymmetries in human body thermal patterns and on detecting appreciable local deviations of skin temperature from the temperature of surrounding tissues [6]. In the present work, we develop the approach first formulated in [7, 8], which offers a more comprehensive view on life processes' thermal representation on human skin, with a special emphasis on the in-depth analysis of thermal heterogeneity of the entire body surface. An assumption was put forward and justified $[7,8]$ that an integrated response from a human organism could be expected even at the initial stage of deviation of physiological functions of this or that organ (or system) from the norm, and the associated changes could be detected and quantified by a computer-aided diagnostic instrument, including focal plane array (FPA) based IRT [9-11]. This basically new approach to medical infrared thermography is worth being studied in more detail. One of the first lines of attacking the problem of quantitative interpretation of heterogeneous thermograms is advanced in the present work.

The mere issue of differentiating between homogeneous and heterogeneous thermograms is still open for discussion. Except for [7, 8, 12], we are not aware of any publications either in the field of IR imaging or in other areas, which would be specially aimed at searching for methods of an objective quantitative analysis of highly heterogeneous thermograms (or other images) with the attention being focused exactly on indicators of heterogeneity.

\section{Approaches to the quantitative description of the degree of thermogram heterogeneity}

One algorithm recently proposed [12] for the quantitative description of heterogeneous thermograms includes spatial differentiation of a discrete image and a subsequent Fourier analysis. The use of this algorithm is based on the general view of the heterogeneous image, which resembles a quasi-periodic structure. This structure, in turn, can be associated with the notion of a texture. A reciprocal of the texture period is used as a quantitative measure of heterogeneity.

Another approach devoid of some bottlenecks of the first one is based on using the Haar wavelet transform. It was also described in [12]. This algorithm allowed the quantitative characteristics of heterogeneity to 
be reconciled with the visual patterns of the actually measured test thermograms arranged in the order of increasing inhomogeneity.

The main challenge in implementation of the intriguing idea of creating an automated IR-imaging diagnostic instrument is to "teach" the computer code:

a) to detect thermal inhomogeneities on the surface important for diagnostic purposes;

b) to determine the quantitative characteristics of inhomogeneity areas;

c) to distribute (sort) the inhomoheneity areas in terms of their spatial and amplitude characteristics (to construct spatial and/or amplitude spectra) for an intellectual analysis of results to be subsequently performed.

These are the issues discussed in the present paper.

\section{Principle of "thermal relief flooding" for searching and identification of hyperthermia foci}

Let us consider an $\mathrm{N} \times \mathrm{M}$ thermogram as a three-dimensional surface ("relief") rather than a twodimensional table of temperatures conventionally converted to a set of halftones or colours. This relief is defined in the form of a table of heights $\mathrm{T}(\mathrm{Xi}, \mathrm{Yj})$, where $\mathrm{T}$ is the temperature; $\mathrm{Xi}$ and $\mathrm{Yj}$ are the pixel coordinates; the subscripts $i$ and $j$ take the values from 1 to $N$ and from 1 to $M$, respectively. This surface can be "flooded by water," namely, all points of this surface with heights lower than a certain numerical value TF (temperatures below a certain value TF) are truncated. The apparent similarity with the known methods of image analysis (e.g., the method of constructing isolines or contour mapping) does not downgrade the significance of the material described, because all details of the approaches and results described here are intimately connected with specific features of IR images.

First, individual vertices of the above-determined 3D surface are found, and their number is calculated. The principle of searching for vertices implies the search for points and flat portions of the surface with no way upward. A possible effect of IR detector noise is taken into account. To identify the spots, it is more convenient to use the process of water drain rather than flooding, in order to find the vertices first and then to track each "island." The process of water drain includes an analysis of the increase in horizontal cross-sectional area of a three-dimensional heat spot $\mathrm{T}(\mathrm{X}, \mathrm{Y})$.

\section{Identification and determination of the characteristic size of local heat spots on the 3D thermogram} by analyzing the growth rate of their sectional area with respect to the overall sectional area

\subsection{Uniform "water drain"}

The study of the specific features of the temperature distribution on the human body surface shows that the heat spots with linear sizes of one or several centimetres are smooth bell-shaped functions in the temperature cross section, which are qualitatively similar to the normal distribution function (Gaussian distribution). In the case of multiple dilatation of surface vessels, such heat spots cross each other and generate a profile resembling a hilly country.

Let us consider an artificial spot in the form of the normal distribution function. The spot is defined as

$$
T=T_{0} e^{-\frac{r^{2}}{2 \sigma^{2}}},
$$

where $\mathrm{T}$ is the excess of temperature above a certain level in the vicinity of this spot, T0 is the excess of temperature at the spot vertex, $r$ is the distance between the vertex and an arbitrary point in the vicinity of the spot, and $\sqrt{2} \sigma$ is the characteristic half-width of the spot. We are going to track the specific features manifested in analyzing consecutive cross sections of this 3D "bell".

During the water drain, the cross-sectional area of the spot gradually increases. Our algorithm of analyzing these changes implies considering the ratio of the increase in the spot area at a certain time step (growth rate) to the current area of this spot. Let us comment why we use this characteristic.

Let us algebraically transform relation (1). The cross-sectional area of the spot is

$$
\begin{aligned}
& S=\pi r^{2}=-2 \pi \sigma^{2} \ln \frac{T}{T_{0}} \\
& \frac{d S}{S}=\frac{d T}{T \ln \frac{T}{T_{0}}}
\end{aligned}
$$

We are interested in the behaviour of relation (2). With a constant step $\mathrm{dT}$, the character of this dependence is determined by the function

$$
F(T)=-\frac{1}{T \ln \frac{T}{T_{0}}}
$$

plotted in figure 1. 
If we move from top to bottom, cutting the spot and storing the values of $T$ and $d S / S$ for each section, the smallest ratio of the growth rate dS to the cross-sectional area $S$ is reached at a temperature T0/e $(e=$ $2.71828 \ldots)$. In this case, the spot radius is $\sqrt{2} \sigma$; hence, it can describe the characteristic cross-sectional area of the spot. Thus, by controlling function (2) and finding its minimum, we can obtain the effective cross-sectional area of the heat spot smoothly distributed over the surface in accordance with a law similar to (1).

For closely located spots, it seems reasonable to restrict the spot size by the saddle point between the neighbouring foci.

\subsection{Expansion of method capabilities by using an individual approach to the analysis of each spot}

We developed a method that ensures an individual approach to the analysis of each spot observed on the thermogram. After the spot-vertex search, the data obtained are refined by analyzing the data for each individual spot with a new (smaller) uniform temperature step specific for each particular spot.

\section{Identification and characterization of inhomogeneities on artificial and real thermograms}

\subsection{Generation of artificial thermograms}

To check the adequacy of operation of the algorithms proposed, we designed and used a generator of artificial thermograms. An artificial thermogram was generated by random arrangement of "Gaussian" spots (1) on a constant-temperature "foundation" (e.g., $25^{\circ} \mathrm{C}$ ). The spot centre was defined by the coordinate pair (X0, Y0). The values of the parameters $\mathrm{XO}, \mathrm{Y} 0, \mathrm{TO}$, and $\sigma$ were varied within certain limits with the use of a random number generator. An additional condition was avoiding of obvious coalescence of spots (too small distance between their centres). For this purpose, we controlled the distance $L$ between the neighbouring vertices. It had to be greater than a prescribed upper limit of the spot radius:

$$
\min (L) \geq \max (\sqrt{2} \sigma)
$$

Field discreteness was taken into account in artificial thermogram generation, which allowed the method sensitivity to the photodetector array dimension to be checked.

In constructing a homogeneous thermogram for one individual, following $[7,8]$, we left the spot centres $(\mathrm{X} 0, \mathrm{YO})$ on the same place and randomly reduced the maximum values of T0 with an appropriate increase in $\sigma$. The value of $\sigma$ was defined by the relation

$$
V=2 \pi T_{0} \sigma^{2}
$$

so that the volume $V$ under the three-dimensional plot $T(r)$ was unchanged, which qualitatively reflected the fact of energy conservation during heat spreading over the surface.

\subsection{Verification of adequacy of computer code operation}

Figure 2 shows a highly heterogeneous (left) and less heterogeneous (right) artificial thermograms. In obtaining the right pattern from the left pattern, we multiplied the value of T0 of an arbitrary spot by the number randomly generated in the interval from 0 and 0.7 .

For the pair of thermograms in the upper row of figure 2, the search and characterization of heat spots were performed by the method of an individual analysis (Sec. 4.2). As a result, with the initial "water drain" with a step $\mathrm{dT}=0.05^{\circ} \mathrm{C}$, we identified 131 (highly heterogeneous thermogram) and 52 (less heterogeneous thermogram) spots out of 142 Gaussian spots randomly distributed over the surface. The shaded areas in figure 2 (middle and lower rows) show hyperthermia fields automatically found on these thermograms by the computer code. After generation of the thermogram shown in figure 2 on the right, some of the original 142 spots transformed to negligibly small perturbations, which could not be detected on the background of other spots.

The missing 142-131=11 spots of heterogeneity can be reasonably explained by interference of the neighbouring temperature "hills," leading to their coalescence (inadequacy of criterion (4)). Note that the human eye in such cases can also distinguish only one local temperature maximum rather than two or more.

\subsection{Identification of "hidden" heat spots}

By careful visual inspection of the middle row of thermograms in figure 2, one can find areas where the spot is visually observed, but it is not detected by the software. Figure 3 shows the temperature distribution in a horizontal section passing through the centre of the "hidden" spot. The difference in temperature between the vertex of the sought spot and the nearest saddle point is less than $0.05^{\circ} \mathrm{C}$. With the initial search step of $0.05^{\circ} \mathrm{C}$, no more than one section of the weak spot can be obtained, and the code recognizes it as noise. 
To increase the probability of detecting low-height spots, we can make the first run more discrete by reducing, for instance, the basic search step from $0.05^{\circ} \mathrm{C}$ to $0.01^{\circ} \mathrm{C}$. The analysis of the thermograms shown in the upper row of figure 2 with a step of $0.01{ }^{\circ} \mathrm{C}$ is illustrated in the lower row. The "hidden" spot is now detected. Thus, the method efficiency is higher than $95 \%$ for the heterogeneous thermogram, which can be considered as a good result.

\subsection{Analysis of real thermograms}

Figure 4 shows the real thermograms of lower extremities, which were obtained in examining one person with different states of his vascular system responsible for obtaining either a fairly homogeneous thermogram (on the left) or a highly heterogeneous thermal pattern (on the right). The thermograms were measured with the TKVrIFP focal plane array based IR system [11] with the array dimension of 128×128 pixels. In addition, for research purposes, the image was digitally increased to $256 \times 256$ pixels, being simultaneously smoothed by the Lagrangian polynomial interpolation over four points (to simplify interpolation, the real size was reduced to $252 \times 252$ pixels). The code was used to analyze both dimensional cases.

The results of code operation are also plotted in the thermograms in the upper and lower rows of figure 4. The shaded areas show the fields found on the thermograms by the method described above. It is seen that the result of code operation with real images, as well as with artificial images, provides a satisfactory description of the true surface distribution and the sizes of visually observed inhomoheneities. As the array dimension is increased, the number of detected spots becomes appreciably greater. It increases from 27 to 59 on thermograms measured before the human organism was subjected to thermal loading and from 36 to 143 after thermal loading which is responsible for a drastic increase in heterogeneity of the temperature pattern. Moreover, digital smoothing allows the shape of some comparatively large spots to be identified more clearly.

Note that the spots were sought on both the original and zoomed-in thermograms with a minimum area of 10 pixels allocated for the spot. This means that the number of small spots detected on the $256 \times 256$ zoomed-in thermogram drastically increases.

\subsection{Spectrum of distribution of temperature heterogeneity spots in terms of their area}

In medical applications, an important issue in the heterogeneity analysis is not only the fact of vessel expansion or the vessel filling by warmer blood. It is also important to know which vessels (small or large) changed their state, which group of vessels will come back to the norm, and which characteristic time will be needed for that. Correspondingly, an important problem is not only obtaining a quantitative integral estimate of thermal pattern heterogeneity, but also automatic sorting of heterogeneity spots in terms of their size. If the latter problem is solved, we would be able to perform an objective study of the state kinetics of individual groups of heat spots.

Figure 5 shows some examples of the spectral distributions of the spot size for data shown in figure 4 . To reach a unified metric scale, the number of pixels on the abscissa axis for the zoomed-in thermogram is divided by four. It is seen from figure 5 that the code provided an adequate statistical treatment of the areas of temperature spots.

\section{Coalescence of heat spots during their spreading and approaching}

One of the elementary processes whose accumulation can alter the subjective estimate of the image is the coalescence of two individual heat spots into one. The associated processes can be physically modelled in the future having regard to the peculiarities of heat propagation in the human body [13-15]. We "asked" the computer code to track the changes in the sizes of two spots approaching each other, which were found by the algorithm described above (Sec. 5).

Figure 6 shows the calculated effective cross-sectional area of the spot as a function of the distance between the vertices of the pair considered. Beginning from the distance equal to 102 pixels, the effective crosssectional area gradually decreases. This is a consequence of direct interaction of the spots with each other, leading, in particular, to deformation of the spot vertex. At this stage, we see a saddle between the spots, which moves upward (toward the narrowing peaks). As a result, the effective cross-sectional area detected by the code is smaller.

Small vertices of the spots finally merge together and form one wide spot. This jump-like process is shown by the dashed straight line in figure 6 . This jump is similar to that observed in our computer heat-spreading experiments which were based on a full-scale [16] experiment. The nonmonotonic behaviour (jumps) of the curve in figure 6 is associated with the image discreteness.

\section{Conclusions}

The present paper describes convincing arguments for considering the degree of heterogeneity of IR images as an independent quantitative characteristic of the surface heat field. Methods of the quantitative description of heterogeneous thermograms are presented. The current status of the problem is analyzed. With a reference for the previous publication [12], it is noted that a possible approach to quantifying heterogeneous 
images may be the use of the Fourier analysis. Another approach devoid of some bottlenecks of the first one, which was also described in [12], is based on the use of the Haar wavelet transforms.

A new approach to solving the problem is described, which includes a direct statistical analysis of the distribution of multiple heat spots with elevated temperature on the surface. This approach is implemented with a specially developed software code. This code allows one to detect both high-temperature and weak-intensity spots on the thermogram, to analyze their shape in a three-dimensional $(X, Y, T)$ representation, to estimate the cross-sectional area of such three-dimensional "hills", to increase the dimension of the discrete image with improving the spot-recognition quality without violating the reliability of experimental data, to compare the found heterogeneities with the original thermal pattern, and finally to construct the spectral characteristics reflecting the distribution of heat spots in terms of their areas over the thermogram field. For medicine, we assume that one of the diagnostically essential quantitative features may be the character of transformation of such spectra with time, which can be different for ill and healthy people.

It can be simultaneously noted that the new quantitative notion "the degree of heterogeneity" and the approaches to solving the problem of the quantitative description of heterogeneity, which were described in this paper, are applicable not only to medical problems, because heterogeneous images obtained by various optical and other devices, including X-ray equipment, are also encountered in many other fields of our life.

The method and corresponding software developed allow the user to distinguish almost all local temperature heterogeneities with arbitrary shapes and amplitudes on the background of the thermogram, the latter being also initially heterogeneous. This feature is an important practical outcome of these activities, because it allows doctors to observe functioning of vessels lying in an immediate vicinity of skin (normally providing a sharp contrast of the temperature response on the surface), as well as functioning of in-depth vessels.

An additional attribute of the code developed within the present activities is the generator of artificial thermograms with prescribed input parameters (known or obtained by means of random generation). Such thermograms may serve as a convenient "field test" for software codes through comparisons between the subjective (visual) detection of thermograms and their objective characteristics (number of spots and their parameters). They can also be used for comprehensive computer simulations of thermal patterns on the surface, in particular, simulations of the processes of approaching and spreading of local hyperthermia spots. The capabilities of dynamic modelling are demonstrated by examples of studying effects associated with heat dissipation along the surface, leading to coalescence of the neighbouring heat spots, and studying transformation of the thermal pattern in the case of approaching of the epicentres of the temperature spots. The results of these two researches allowed us to discover the threshold character of coalescence of the neighbouring spots, which accompanies the transformation of a heterogeneous thermogram to a diffuse (more homogeneous) one.

The number and shape of heat spots detected by the method proposed were found to be in good agreement (95\%) with the initial parameters of spots defined in modelling artificial heterogeneous thermograms, despite the fact that the spots merged due to their interference had no longer the initial "Gaussian" form. An even more convincing and important result, which proves the possibility of advancing toward an adequate automated analysis of biological and medical thermal images, is the reasonable agreement of results obtained by this method with the visual detection of heterogeneity spots on real thermograms of the human body surface.

As the proposed method of searching for temperature spots is based on the properties of the normal distribution function (see relation (2)), the latter result may be of fundamental importance. It serves as an argument for the fact that the temperature distribution in cross sections of even asymmetric spots of hyperthermia on human skin predominantly obeys the normal distribution law. It is known that the nature has many versatile phenomena described by the normal distribution function. The result of the present work is another example of this law validated experimentally.

Evaluation of the degree of heterogeneity of medical images, combined with the possibility of its quantitative comparisons, may provide valuable information on the physiological status of the organism [7, 8] and substantially expand the capabilities of medical diagnostic methods. For this reason, it is important to find a correlation between the degree of heterogeneity of the temperature pattern and the state of the organism, in particular, the state of its cardiovascular system. Determining the quantitative characteristics of heterogeneity of thermal images substantially expands the prospects of biological and medical IR diagnostics and monitoring, thus, enhancing the method of IR imaging toward automated recognition of deviations of the organism from normal functioning.

\section{Acknowledgement}

The authors are grateful to A. F. Sapetina for her assistance in modelling the process of spreading of heat spots. This work was supported by the Siberian Branch of the Russian Academy of Sciences (Integration Project No. 46-2006).

\section{REFERENCES}

[1] H. Lecerof, S. Bornmyr, B. Lilja, G. De Pedis, and U. L. Hulthen. Acute effects of doxazosin and atenolol on smoking-induced peripheral vasoconstriction in hypertensive habitual smokers, J. Hypertens. Suppl., 8 (1990) S29-S33.

[2] T. C. Tham, B. Silke, and S. H. Taylor. Comparison of central and peripheral haemodynamic effects of dilevalol and atenolol in essential hypertension, J. Hum. Hypertens., 4 (1990) 77-83. 
[3] G. Parrinello, S. Paterna, P. Di Pasquale, A. Pinto, A. Cardinale, T. Maniscalchi, C. Cottone, G. Follone, A. Tuttolomondo, P. Bologna, D. Colomba, A. D'Angelo, A. Ortoleva, L. Garofalo, G. Piovana, E. Capodieci, A. Bova, A. Giubilato, and G. Licata. Effect of subcutaneous sumatriptan on head temperature in migraines, Drugs Exp. Clin. Res., 24 (1998) 197-205.

[4] H. Fushimi, T. Inoue, Y. Yamada, Y. Matsuyama, M. Kubo, and M. Kameyama. Abnormal vasoreaction of peripheral arteries to cold stimulus of both hands in diabetics, Diabetes Res. Clin. Pract., 32 (1996) 55-59.

[5] K. Mabuchi. Clinical significance of thermography - a non-invasive and non-contact method to evaluate peripheral circulatory function in the diagnosis of diabetic complications, Nippon Rinsho, 48 (1990) 580-587.

[6] Basics of Clinical Remote Thermodiagnostics. Ed. by L.G.Rozenfel'd. Kiev, Zdorov'ya (1988).

[7] B. G. Vainer. FPA-based infrared thermography in physiology: Investigation of vascular response, perspiration, and thermoregulation in humans. Novosibirsk, Publishing House SB RAS (2004) (in Rus.)

[8] B. G. Vainer FPA-based infrared thermography as applied to the study of cutaneous perspiration and stimulated vascular response in humans, Phys. Med. Biol., 50 (2005) R63-R94.

[9] A. Manissadjian, P. Tribolet, P. Chorier, and P.Costa. Sofradir infrared detector products: the past and the future, Proc. SPIE, 4130 (2000) 480-495.

[10] R. D. Ecker, S. J. Goerss, F. B. Meyer, A. A. Cohen-Gadol, J. W. Britton, and J. A. Levine. Vision of the future: initial experience with intraoperative real-time high-resolution dynamic infrared imaging. Technical note, J. Neurosurg., 97 (2002) 1460-1471.

[11] G. L. Kurishev, A. P. Kovchavtzev, B. G. Vainer, A. A. Guzev, V. M. Bazovkin, A. S. Stroganov, I. M. Subbotin, I. M. Zakharov, V. M. Efimov, K. O. Postnikov, I. I. Lee, N. A. Valisheva, and Z. V. Panova. Medical infrared imaging system based on a $128 \times 128$ focal plane array for $2.8-3.05 \square \mathrm{m}$ spectral range, Optoelectronics, Instrumentation and Data Processing (Autometria), 4 (1998) 5-10.

[12] M. S. Tarkov, and B. G. Vainer. Evaluation of a thermogram heterogeneity based on the wavelet Haar transform, In: Proc. IEEE Int. Siber. Conf. on Control and Communications (SIBCON-2007), Tomsk, The Tomsk IEEE Chapter \& Student Branch (2007) 145-152.

[13] H. H. Pennes Analysis of tissue and arterial blood temperature in the resting human forearm, J. Appl. Physiol., 1 (1948) 93-102.

[14] B. Vainer, P. Belozerov, and V. Baranov. Use of IR thermography for bioheat transfer studies, In: Proc. 7th Intern. Conf on Quantitative IR Thermography, July 5-8, 2004, von Karman Inst. for Fluid Dynamics, RhodeSt-Genese, Belgium (2004) J.8.1-J.8.4.

[15] Z. Wu, H. H. Liu, L. Lebanowski, Z. Liu, P. H. Hor. A basic step toward understanding skin surface temperature distributions caused by internal heat sources, Phys. Med. Biol., 52 (2007) 5379-5392.

[16] B. G. Vainer. Medicine-applicable non-steady-state phenomena inspection through the use of infrared thermography, In: Quantitative InfraRed Thermography 5, Eurotherm Seminar 64, QIRT'2000, Reims, France, July 18-21, 2000. Proceedings. Ed. by D.Balageas, J.-L.Beaudoin, G.Busse, and G.M.Carlomagno. UTAP URCA (2000) 405-408.

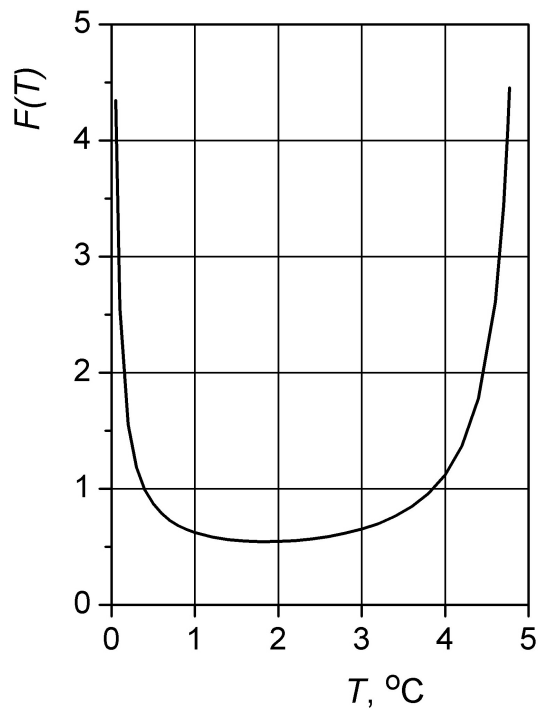

Fig. 1. Function (3) plotted for $T_{0}=5{ }^{\circ} \mathrm{C}$ corresponding here to the height of the local "heterogeneity hill" on the thermogram above the constant-temperature "foundation" 


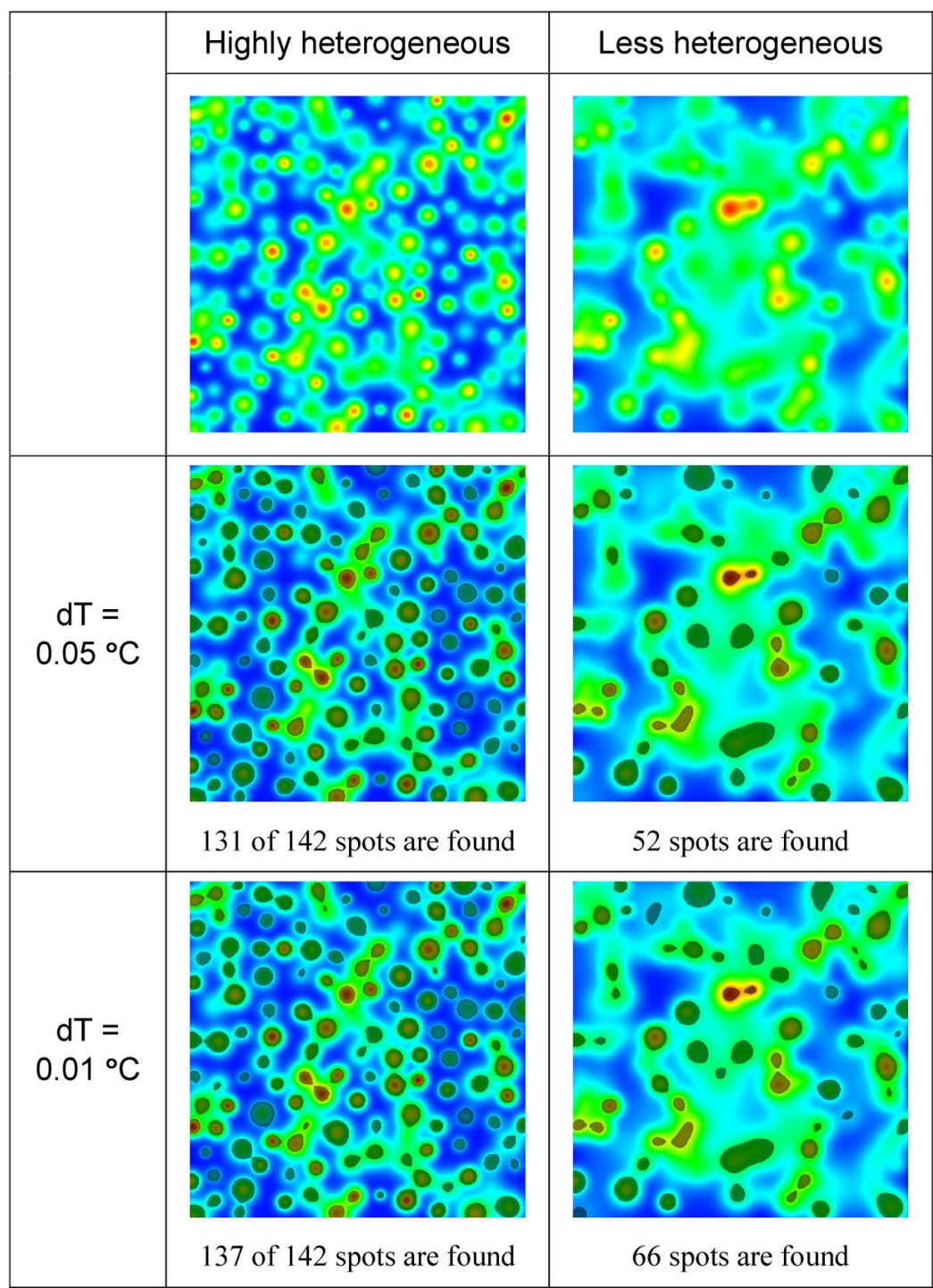

Fig. 2. Highly heterogeneous (left) and more diffuse (right) thermograms
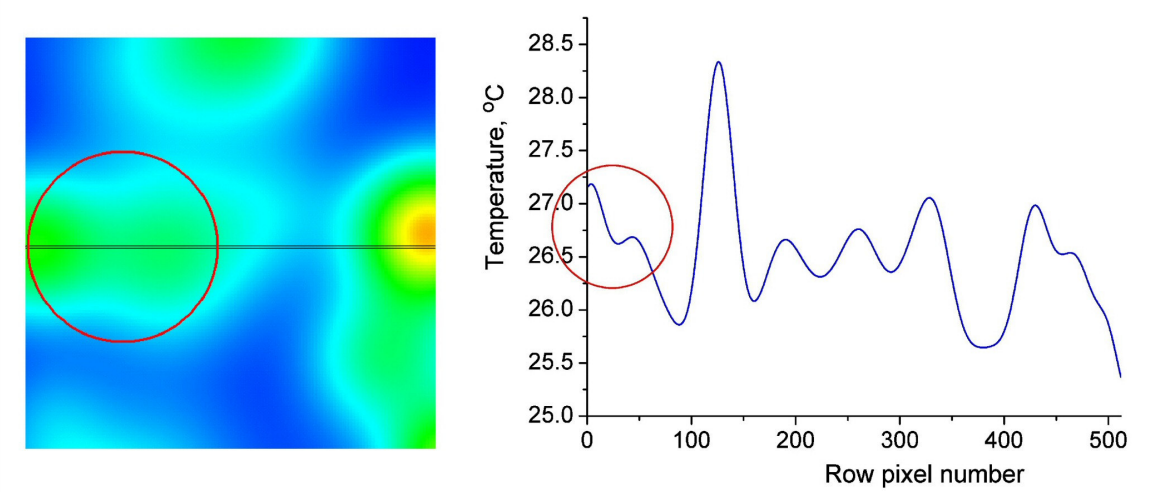

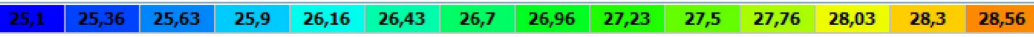

Temperature scale, ${ }^{\circ} \mathrm{C}$

Fig. 3. A "hidden" heat spot 


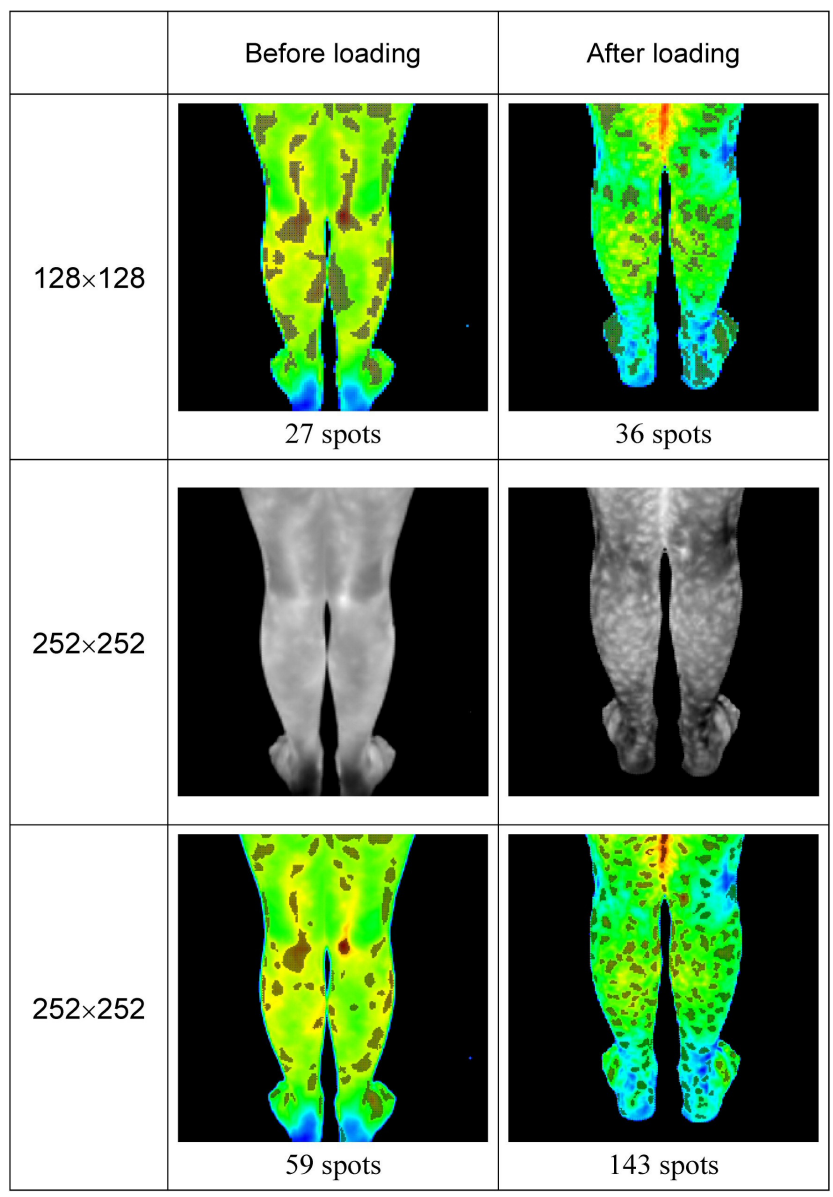

Fig. 4. Thermograms of the same person, measured before (left) and after (right) thermal loading of his organism

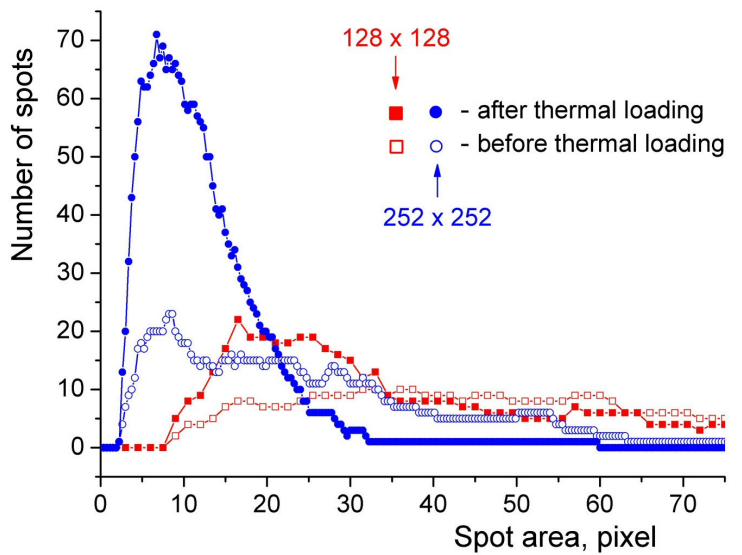

Fig. 5. Spectral distributions of heat spots in terms of their areas: results of an automated analysis of thermograms shown in figure 4 . The data are given for the initially measured $(128 \times 128)$ and artificially expanded (252×252) thermograms. The open and filled symbols are the results obtained before and after thermal loading of the organism

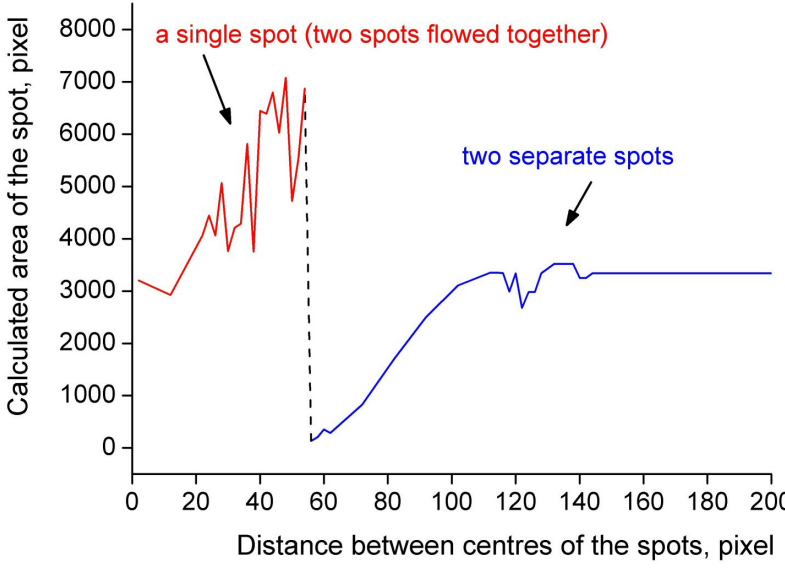

Fig. 6. Cross-sectional area of heat spots versus the distance between their vertices while modeling the process of approaching of these spots 